\title{
Interactions in Polar Media. II. Continua*
}

\author{
R. A. Marcus $\dagger$ \\ Departments of Chemistry, Brookhaven National Laboratory, Upton, New York, and Polytechnic Institule of Brooklyn, Brooklyn 1, Nezw York
}

(Received 17 January 1963)

\begin{abstract}
It is shown that the electronically polarizable continuum model of a particle satisfies the equations of Part I, after suitable choice of operator $A_{i}$ appearing there. The proof is given for the case where the system is regarded as composed of particles and treated statistically mechanically. It is also given for the case where several particles receive special attention and the remainder of the system (the "medium") is treated as an orientationally and electronically polarizable continuum. For the second case it was necessary to extend the results of Part I, so as to include several particles in the presence of the above "medium" and to compute the free energy of such systems. Calculations are given for media possessing equilibrium and nonequilibrium dielectric polarization.

It follows from the foregoing proofs that a wide variety of models assumed in the literature for treating polar interactions are special cases of the model in Part $I$ and of the extension to particle-medium systems in this paper. Electrode systems, for example, are included, even when the electrode is treated in the usual dielectric continuum manner.

The relation and relative merits of the two models for the induced charge distribution that are standard in the literature, both special cases of Part I, are discussed. These models are the induced dipole and the electronically polarizable continuum. Possible direct experimental investigation of the second of these by scattering experiments is examined.
\end{abstract}

\section{INTRODUCTION}

$\mathbf{I}^{\mathrm{N}}$ Part $\mathrm{I}^{1}$ equations were derived for the polar contribution to the potential energy of interaction between particles. Each particle represented a single molecule or any collection of molecules (a whole electrode, for example) at any specified nuclear configuration. The equations involved multipolar permanent and induced charge distributions on the particles and constituted a generalization of earlier potential energy expressions $\mathrm{s}^{2}$ in which the particles were restricted to being single molecules interacting via permanent and induced dipoles only. One use of the equations was in the formulation of theories of phenomena related to polar interactions, using fewer assumptions and applicable to a wide class of more specialized models commonly found in the literature. Some of these applications are mentioned later.

In the present paper it is shown that the equations of Part $I$ are also formally satisfied by a common model of a particle, wherein it is treated as an electronically polarizable dielectric continuum containing some or no fixed charge distribution..$^{3-8}$ One merely chooses an

\footnotetext{
* Research performed in part under the auspices of the $U . S$. Atomic Energy Commission.

$\dagger$ Alfred P. Sloan Fellow. Visiting Senior Scientist, B.N.L. Present address: Polytechnic Institute of Brooklyn.

IR. A. Marcus, J. Chem. Phys. 38, 1335 (1963).

M. Mandel and P. Mazur, Physica 24, 116 (1958).

${ }^{3}$ H. Frohlich, Theory of Dielectrics (Oxford University Press, London, 1950)

${ }^{4}$ T. G. Scholte, Physica 15, 439 (1949); cf. Ref. 9.

$5 \mathrm{~J}$. G. Kirkwood in Proteins, Amino Acids and Peptides

(Reinhold Publishing Corporation, New York, 1943).

${ }^{6}$ J. G. Kirkwood, J. Chem. Phys. 2, 351 (1934)

7 F. H. Westheimer and J. G. Kirkwood, J. Chem. Phys. 6, 505,515 (1938)

${ }^{8}$ See, e.g., H. C. van de Hulst, Light Scattering by Small Pariicles (John Wiley \& Sons, Inc, New York, 1957).
}

appropriate function for the operator $A_{i}$ appearing in Part I. (An important example of such a model occurs in the standard electrostatic treatment of polar interactions between electrodes and particles in solution: The electrode is regarded as being a continuum of infinite dielectric constant.) Accordingly, any statistical mechanical theory of a phenomenon developed from the interparticle potential energy expression of Part I must also be satisfied by this idealized electronically polarizable continuum model of a particle. We make use of this result elsewhere.

Because of computational difficulties in the statistical mechanical treatment of positions of the nuclei, many literature calculations are based on a continuum treatment of part of the orientation and electronic polarization in the system. Several particles of specified nuclear configuration receive special attention and the remainder of the system (the "medium") is treated as an orientationally and electronically polarizable dielectric continuum. The particles have either been treated as having some permanent and induced dipolar charge distribution ${ }^{3,9}$ (i.e., as being a special case of the charge distribution in Part I) or as having a permanent charge distribution and as being the electronically polarizable continuum mentioned earlier. ${ }^{3-7}$ (Henceforth, for purposes of this paper only, the words "orientationally polarizable continuum" and "medium" are used interchangeably. In a statistical mechanical treatment, the totality of molecules of the medium can be treated as a single particle, ${ }^{1}$ and such a system is called here a "particles-only system.") Using the model of the particles given in Part $I$, the polar con-

${ }^{9}$ C. J. F. Böttcher, Theory of Electrical Polarization (Elsevier Publishing Company, Inc., New York, 1952). 
tribution to the free energy of these systems is calculated in the present paper for any specified nuclear configuration of the particles. It is shown that the electronically polarizable continuum model of a particle is also a special case of the molecular model of Part $I$ in a medium-particle system (as it is in the purely particle system discussed earlier). The proof is given first for the case where the orientation part of the dielectric polarization of the medium is in electrostatic equilibrium with the charge distribution on the particles and then for the case where it is not. ${ }^{10}$ The electronic part of the polarization of the medium is taken to be that dictated by the particles' charges and the medium's orientation polarization. ${ }^{10}$

The relation between the two standard literature models of the induced charge distribution (both special cases of that in Part I), i.e., the induced dipolar and the electronically polarizable continuum models, is discussed in a concluding section of this paper. Also discussed is the possibility of direct investigation of the continuum model of a gaseous molecule by ion scattering experiments.

Each of the two specialized models has its merits, the induced dipole approximation being more appropriate for small molecules and the electronically polarizable continuum model being suited to very large ones, a crystal for example. In particular, the method of approximating an induced charge distribution by its leading term, the induced dipole, does not explain the dissymmetry of light scattering by large particles. ${ }^{8}$ However, the dissymmetry is explained by the continuum model, because of the appearance of higher terms than the dipolar one. ${ }^{8}$ Moreover, even a uniform static field sometimes produces induced moments higher than the dipole one. Lack of knowledge of these higher moments has encouraged the use of the continuum model. In the case of spheres and, for the dipolar term under certain conditions, ellipsoids, the two models prove to yield identical results in a uniform field, provided the dielectric susceptibility of the continuum is properly chosen. The choice of this susceptibility is described.

Elsewhere, application is made of the equations of Part I to a statistical-mechanical formulation of theories involving polar interactions in condensed phases and of the equations of the present paper to a formulation based on the continuum treatment of the medium. Several problems to be treated involve nonequilibrium dielectric polarization of the medium and deal with electron transfer rates in solution and at electrodes, and with shifts and broadening of electronic spectral bands of polar solutes by polar solvents. ${ }^{11}$

${ }^{10}$ For a description of nonequilibrium polarization systems $\mathrm{cf}$. R. A. Marcus, J. Chem. Phys. 24, 979 (1956) and Ref. 11. Such systems play a role in electron transfer processes and in spectral shifts of polar solutes caused by polar solvents.

11 R. A. Marcus, J. Chem. Phys. 38, 1858 (1963).

\section{MOLECULAR TREATMENT OF PARTICLES}

As in Part I let $\phi_{i}(\mathbf{r})$ be the electrostatic potential arising from all parts of the system other than from the charge density $\rho_{i}(\mathbf{r})$ on particle $i$. An equation of Part I for $\phi_{i}$ is easily extended to the case where, besides the particles, a medium is present having a polarization $\mathbf{P}(\mathbf{r})^{1,10,12}$ :

$$
\phi_{i}\left(\mathbf{r}^{\prime}\right)=\sum_{j \neq i} \int \frac{\rho_{j}(\mathbf{r}) d \mathbf{r}}{r}+\int \mathbf{P}(\mathbf{r}) \cdot \nabla r^{-1} d \mathbf{r}
$$

where integration is over the entire volume of the system, $1 / r$ denotes $1 /\left|\mathbf{r}-\mathbf{r}^{\prime}\right|, \mathbf{P}(\mathbf{r})$ vanishes inside the region $V_{i}$ occupied by any particle $i$, and $\rho_{i}(\mathbf{r})$ is given by (2). [To obtain the equations of Part I, P(r) is set equal to zero throughout this paper.]

$$
\rho_{i}(\mathbf{r})=\rho_{i}{ }^{0}(\mathbf{r})+\rho_{i}{ }^{\text {in }}(\mathbf{r}),
$$

where $\rho_{i}^{0}$ is the charge distribution on $i$ when it is isolated. ${ }^{13,14}$ The induced charge distribution $\rho_{i}{ }^{\text {in }}$ is related to $\phi_{i}$ via a linear Hermitian operator ${ }^{1} A_{i}$ :

$$
\rho_{i}^{\text {in }}(\mathbf{r})=A_{i}\left(\mathbf{r}, \mathbf{r}^{\prime}\right) \phi_{i}\left(\mathbf{r}^{\prime}\right) .
$$

When only particles are present, each having a specified nuclear configuration, the polar contribution to the interparticle potential energy $U^{\mathrm{pol}}$ is given by (4). ${ }^{1}$

$$
U^{\mathrm{pol}}=\frac{1}{2} \sum_{i} \int \phi_{i} \rho_{i}{ }^{0} d \mathbf{r}
$$

where $\phi_{i}$ is given by (1) with $\mathbf{P}=0$.

When both particles and the polarized medium are present, it is the polar contribution to the free energy

${ }^{12}$ Cf. M. Mason and W. Weaver, The Electromagnetic Field (The University of Chicago Press, Chicago, 1929), p. 67; cf. G. Joos, Theoretical Physics (Blackie and Son, Ltd., London, 1934), p. 267.

${ }^{13} \rho_{i}{ }^{0}$ is the sum of a continuous function due to the electrons of $i$ and of $\delta$ functions (of suitable strength) due to the nuclei of $i$. For an ion of charge $q, \rho_{i}{ }^{0}$ is sometimes approximated by a $\delta$ function to represent a point charge $q$ situated at $\mathbf{r}_{0}, \rho_{i}{ }^{0}(\mathbf{r})=q \delta\left(\mathbf{r}-\mathbf{r}_{0}\right)$. A polar molecule of dipole moment $\mu$ is often treated as a dipole, and higher moments are ignored. This approximation corresponds ${ }^{14}$ to $\rho_{i}{ }^{0}=-\mu \cdot \nabla, \delta\left(\mathbf{r}-\mathbf{r}_{0}\right)$. Higher multipoles correspond in the same way to higher derivatives of the $\delta$ function. Sometimes a molecule is regarded as consisting of several separated point charges, so the corresponding $\rho_{i}{ }^{0}$ is the sum of several $\delta$ functions.

Any such approximations are special cases of the present more general formulation and are therefore automatically included in it. Various equations in the literature can be obtained from those of this paper by noting that if $\phi$ is any function continuous at $\mathbf{r}_{0}$, then $\int \phi \rho_{i}{ }^{0} d \mathbf{r}$ equals $q \phi\left(\mathbf{r}_{0}\right)$ and $\mu \cdot\left(\nabla_{\phi}\right)_{r 0}$ for the two examples cited above. [Use is made of

$$
\delta\left(\mathbf{r}-\mathbf{r}_{0}\right)=\delta\left(x-x_{0}\right) \delta\left(y-y_{0}\right) \delta\left(z-z_{0}\right)
$$

and of any integration by parts in the second example.]

In the literature $\rho_{i}$. in is invariably approximated by an induced dipole, $\alpha E$; i.e., $\rho^{\text {iv }}(\mathbf{r})=-\alpha E\left(\mathbf{r}_{0}\right) \cdot \nabla_{r} \delta\left(\mathbf{r}-\mathbf{r}_{0}\right)$, where $\alpha$ is the polarizability tensor and $E$ the field arising from the other parts of the system. [See Eqs. (40) and (41) of Ref. 1.]

${ }_{14} \mathrm{Cf}$. discussion of derivatives of $\delta$ functions by B. Friedman in Principles and Techniques of Applied Mathematics (John Wiley \& Sons, Inc., New York, 1956). 
$F^{\text {pol }}$ which is of interest. When $\mathbf{P}(\mathbf{r})$ is in electrostatic equilibrium with the charge equilibrium, e.g., when it is given by $-\chi(\mathbf{r}) \nabla \psi(\mathbf{r})$, where $\chi$ is the dielectric susceptibility of the medium and $\psi$ is the electrostatic potential arising from all parts of the system, then $F^{\mathrm{pol}}$ is given by the rhs of (4), with $\phi_{i}$ given by (1). A straightforward proof of (4) for $U^{\mathrm{pol}}$ in the particleonly case, and for $F^{\text {pol }}$ in the particle-medium one, can be given simultaneously. In both cases it equals the work needed to charge every $\rho_{i}{ }^{0}$ from 0 to its final value, $\rho_{\imath}{ }^{0}{ }^{15}$ This proof supplements the two independent proofs given in Part I for $U^{\mathrm{pol}}$. The value of $F^{\mathrm{pol}}$ for a particle-medium system when $\mathbf{P}(\mathbf{r})$ is not in electrostatic equilibrium with the $\rho_{i}$ is given later.

Equations (1) to (4) and an equation for $\mathbf{P}(\mathbf{r})$ are the basic equations. We also make use of Eqs. (5) and (6) for the electrostatic potential $\psi(\mathbf{r})$ due to all sources and for the potential $\psi_{\text {is }}(\mathbf{r})$ in a system containing an isolated particle $i$. (This isolated system is denoted by "is.")

$$
\begin{aligned}
\psi\left(\mathbf{r}^{\prime}\right) & =\sum_{i} \int \frac{\rho_{i}(\mathbf{r})}{r} d \mathbf{r}+\int \mathbf{P}(\mathbf{r}) \cdot \nabla r^{-1} d \mathbf{r} \\
& =\phi_{i}\left(\mathbf{r}^{\prime}\right)+\int \frac{\rho_{i}(\mathbf{r})}{r} d \mathbf{r} \\
\psi_{\text {is }}\left(\mathbf{r}^{\prime}\right) & =\int \frac{\rho_{i}^{0}(\mathbf{r})}{r} d \mathbf{r} .
\end{aligned}
$$

\section{ELECTRONICALLY POLARIZABLE CONTINUUM MODEL OF A PARTICLE}

Each particle $i$ is treated as a region having electronic dielectric susceptibility $\chi_{i}(\mathbf{r})$ and having a fixed charge density $\rho_{i}{ }^{v}(\mathbf{r})$. Both $\chi_{i}(\mathbf{r})$ and $\rho_{i}{ }^{v}(\mathbf{r})$ can vary with position $r$ inside the molecule $i$, and both vanish outside $V_{i}$, the volume occupied by $i$. The potential $\psi\left(\mathbf{r}^{\prime}\right)$ is the sum of contributions of the charge distributions $\rho_{i}{ }^{v}$ and from all polarized volume elements, those inside each $V_{i}\left(-\chi_{i} \nabla \psi\right)$ and those outside:

$\psi\left(\mathbf{r}^{\prime}\right)=\sum_{i} \int\left\{\frac{\rho_{i}^{v}}{r}-\chi_{i} \nabla \psi \cdot \nabla r^{-1}\right\} d \mathbf{r}+\int \mathbf{P}(\mathbf{r}) \cdot \nabla r^{-1} d \mathbf{r}$,

$$
\begin{aligned}
& { }^{15} \text { Both } U^{\text {pol }} \text { and } F^{\text {pol }} \text { equal } \\
& \qquad \sum_{i} \int_{\mathbf{r}} \int_{\rho_{i} 0=0}^{\rho_{i}=\rho_{i}} \phi_{i} d \rho_{i}{ }^{0}(\mathbf{r}) d \mathbf{r},
\end{aligned}
$$

$\phi_{i}$ in each case being given by the appropriate expression. Since $\phi_{i}$ depends linearly on $\rho_{i}^{0}$, this equation can be integrated to yield (4), as follows: Let $\lambda$ be a charging parameter which increases from 0 to 1 , and let the values of $\phi_{i}, \psi, \rho_{i}{ }^{0}, \psi_{i \text { a }}$ during charging be denoted by a subscript $\lambda$, i.e., $\rho_{i}{ }^{\lambda}{ }^{\lambda}=\lambda \rho_{i}{ }^{0}$, so that the $d \rho_{i}{ }^{0}$ above becomes $d \rho_{i}{ }^{0 \lambda}$, i.e., $\rho_{i}{ }^{0} d \lambda$. It may be seen from Eqs. (1)-(6), after division by $\lambda$ and using the linearity of $A_{i}$, that the triplet $\left(\phi_{i}{ }^{\lambda} / \lambda, \psi^{\lambda} / \lambda\right.$, and $\left.\psi_{i^{\lambda}}{ }^{\lambda} / \lambda\right)$ satisfies the same equations as does $\left(\phi_{i}, \psi\right.$, and $\left.\psi_{\mathrm{ig}}\right)$. Therefore, $\phi_{i}{ }^{\lambda}=\lambda \phi_{i}, \psi^{\lambda}=\lambda \psi, \psi_{\mathrm{ig}}{ }^{\lambda}=\lambda \psi_{\mathrm{is}}$, by a uniqueness theorem. Introduction of this value of $\phi_{i}{ }^{\lambda}$ and $d \rho_{i}{ }^{{ }^{\lambda}}$ leads to $(4)$. where $\mathbf{P}(\mathbf{r})$ vanishes inside each $V_{i}$. For generality, both $\chi_{i}(\mathbf{r})$ and $\chi(\mathbf{r})$ will be regarded as piecewise continuous functions, ${ }^{16}$ though in practice they are usually treated as a special case of these functions, namely piecewise constant ones. The density $\rho_{i}^{v}(\mathbf{r})$ is frequently approximated by certain functions, ${ }^{13}$ but this need not be done here.

For an isolated $i$ we have:

$$
\psi_{\text {is }}\left(\mathbf{r}^{\prime}\right)=\int \frac{\rho_{i}{ }^{v}}{r} d \mathbf{r}-\int \chi_{i} \nabla \psi_{\text {is }} \cdot \nabla r^{-1} d \mathbf{r}
$$

From Eq. (8) the charge density of an isolated $i \rho_{\text {is }}$ is found to be given by (9).$^{17,18}$ Similarly, from Eq. (7) the charge density of an $i$ in the actual system, $\rho_{i}$ is given by (10).

$$
\begin{gathered}
\rho_{\mathrm{is}}(\mathbf{r})=\rho_{i}{ }^{v}(\mathbf{r})+\nabla \cdot\left(\chi_{i} \nabla \psi_{\mathrm{is}}\right)-\delta\left(n_{i}\right) \chi_{i}\left(\partial \psi_{\mathrm{is}} / \partial n_{i}\right), \\
\rho_{i}(\mathbf{r})=\rho_{i}{ }^{v}(\mathbf{r})+\nabla \cdot\left(\chi_{i} \nabla \psi\right)-\delta\left(n_{i}\right) \chi_{i}\left(\partial \psi / \partial n_{i}\right),
\end{gathered}
$$

where $n_{i}$ is the coordinate normal to the surface $S_{i}$ enclosing $V_{i}$, its positive direction being outward from $V_{i}$. We let $n_{i}$ equal zero at $S_{i}$, i.e., $\delta\left(n_{i}\right)$ is a $\delta$ function peaked at $S_{i}$. In the last term $\chi_{i}$ is the limit of $\chi_{i}(\mathbf{r})$ as one approaches $S_{i}$ from within $V_{i}$. [The limit of $\chi_{i}(\mathbf{r})$ as one approaches $S_{i}$ from outside of $V_{i}$ is zero.]

$U^{\mathrm{pol}}$ or $F^{\mathrm{pol}}$ is obtained by subtracting the reversible work to charge the isolated $i$ 's from that to charge

${ }^{16}$ For example, in any bounded region of three-dimensional
pace, a piecewise continuous function has discontinuities at
only a finite number of points, lines, or surfaces.
17 For example, using Green's first identity, the rhs of (8) can be rewritten as

$$
\int \frac{p_{i}{ }^{v}}{r} d \mathbf{r}+\int \frac{\nabla \cdot\left(\chi_{i} \nabla \psi_{\text {is }}\right)}{r} d \mathbf{r}-\int \nabla \cdot\left(\frac{\chi_{i} \nabla \psi_{\text {is }}}{r}\right) d \mathbf{r}
$$

and the third of these integrals equals the surface integral (Gauss' theorem, e.g., Ref. 12),

$$
\int \chi_{i} \frac{\partial \psi_{i s}}{\partial n_{i}} r^{-1} d S
$$

which in terms of a $\delta$ function is

$$
\int \chi_{i} \frac{\partial \psi_{i s}}{\partial n_{i}} r^{-1} \delta\left(n_{i}\right) d \mathbf{r}
$$

$\psi_{\mathrm{is}}$ then becomes

$$
\int\left[\rho_{i}{ }^{v}+\nabla \cdot \chi_{i} \nabla \psi_{i s}-\chi_{i} \frac{\partial \psi_{\mathrm{is}}}{\partial n_{i}} \delta\left(n_{i}\right)\right] d \mathbf{r} / r
$$

The term in [ ] represents $\rho_{\text {is }}$, for $\psi_{\text {is }}$ also equals $\int \rho_{\text {is }} d \mathbf{r} / r$.

$18 \mathrm{O}$. D. Kellogg, Foundations of Potential Theory (Dover Publications, Inc., New York, 1953). 
them in the actual system. $U^{\mathrm{pol}}$ applies to the particlesonly system and $F^{\mathrm{pol}}$ to the particle-medium system.

$$
\begin{aligned}
U^{\mathrm{pol}}(\text { or } F \mathrm{pol})=\sum_{i} \iint_{\rho_{i^{v}}=0}^{\rho_{i}^{v}=\rho_{i}^{v}} \psi d \rho_{i}^{v}(\mathbf{r}) d \mathbf{r} \\
\\
\quad-\sum_{i} \iint_{\rho_{i}^{v}=0}^{\rho_{i}^{v}=\rho_{i}^{v}} \psi_{\text {is }} d \rho_{i}^{v}(\mathbf{r}) d \mathbf{r} .
\end{aligned}
$$

Equation (11) may be integrated, using arguments similar to those used before ${ }^{15}$ but based on the equations of this section. We find

$$
U^{\mathrm{pol} 1}\left(\text { or } F^{\mathrm{pol}}\right)=\frac{1}{2} \sum_{i} \int\left(\psi-\psi_{\mathrm{is}}\right) \rho_{i}{ }^{v} d \mathbf{r}
$$

\section{ELECTRONICALLY POLARIZABLE CONTINUUM MODEL SATISFIES EQUATIONS (1)-(4)}

It is shown now that this model satisfies Eqs. (1)-(4) with special choice of $A_{i}$. Necessary conditions for it to satisfy these equations are:

(i) $\rho_{i \text { ig }}$ and $\rho_{i}$, given by $(9)$ and (10), also equal $\rho_{i}^{0}$ and $\rho_{i}$ in (2) and (3), respectively. (These functions then include a $\delta$ function peaked at $S_{i}$ if $\chi_{i}$ is discontinuous at $S_{i}$.)

(ii) $\psi_{\text {is }}$ and $\psi$, given by (8) and (7), equal those given by (6) and (5), respectively.

(iii) Identical values for $F^{\mathrm{pol}}$ (or $\left.U^{\mathrm{pol}}\right)$.

We use (i) and (ii) to show that one can find a molecular description with a $\rho_{i}{ }^{0}$ and an $A_{i}$ expressed entirely in terms of continuum properties of $i$, i.e., in terms of $\chi_{i}(r), \rho_{i}{ }^{v}$, and geometry of $V_{i}$, and (a crucial point in the argument) independent of the presence of any other $j(\neq i)$, of any external field and of the value of $\chi$. These equations for $\rho_{i}{ }^{0}$ and $A_{i}$ are necessary consequences of this form of equivalence of the two models. It is then shown that the equations are also sufficient, i.e., that (i), (ii), and (iii) can be obtained from them. One may then conclude that a particular molecular description has been found which is mathematically equivalent to the electronically polarizable continuum model of this molecule insofar as polar interactions are concerned, i.e., that Eqs. (1)-(4) are satisfied by this continuum model.

We treat the particles-only and particles-medium systems simultaneously; in the former the symbol $\mathbf{P}(\mathbf{r})$ is to be omitted. We obtain first the central equation, Eq. (16), from which $A_{i}$ for the model may then be deduced.

Equations (2), (5b), and (6) for the molecular model yield

$$
U\left(\mathbf{r}^{\prime}\right)=\phi_{i}\left(\mathbf{r}^{\prime}\right)+\int \frac{\rho_{i}^{\text {in }}}{r} d \mathbf{r}
$$

where

$$
U=\psi-\psi_{\text {is. }}
$$

Subtracting (9) from (10) to obtain $\rho_{i}^{\text {in }}$, inserting into $\int \rho_{i}{ }^{\text {in }} d \mathbf{r} / r$, then reversing steps analogous to those in Footnote 17, and finally inserting into (13), (13) becomes

$$
\phi_{i}\left(\mathbf{r}^{\prime}\right)=U\left(\mathbf{r}^{\prime}\right)+\int \chi_{i} \nabla U \cdot \nabla r^{-1} d \mathbf{r} .
$$

Equation (15) is solved for $U(\mathbf{r})$ in terms of $\phi_{i}$ in Appendix I. [See Eq. (A1) with $Y$ and $W$ replaced by $\phi_{i}$ and $U$, respectively.] From (3), (13), and (A2), with $X$ and $W$ thus replaced, we obtain

$$
\int \frac{A_{i} \phi_{i}}{r} d \mathbf{r}=-\int \phi_{i} \chi_{i} \frac{\partial G_{i}}{\partial n_{i}} d S_{i}+\int \phi_{i} \nabla \cdot\left(\chi_{i} \nabla G_{i}\right) d \mathbf{r},
$$

where $G_{i}\left(\mathbf{r}, \mathbf{r}^{\prime}\right)$ is the Green's function of Eq. (A3) in Appendix I. $G_{i}\left(\mathbf{r}, \mathbf{r}^{\prime}\right)$ depends only on $x_{i}$ and on the geometry of $i$.

Equation (16) is solved for $A_{i}$ in Appendix II. $A_{i}$ is found there to be a linear operator dependent only on the properties of $i$ (specifically on $\chi_{i}$ and on the geometry of $i$ ), and not on the other species, the applied fields, or the medium. In Appendix III, the $\rho_{i}{ }^{0}$ for this model is shown also to depend only on $\rho_{i}{ }^{v}, \chi_{i}$, and geometry of $i$.

Using these equations for $A_{i}$ and $\rho_{i}{ }^{0}$, conditions (i), (ii), and (iii) are then verified in Appendices IV to VII, and proof is now complete.

\section{SYSTEMS WITH NONEQUILIBRIUM POLARIZATION}

We consider now the case where the polarization of the medium $\mathbf{P}(\mathbf{r})$ in a particle-medium system is not in electrostatic equilibrium with the $\rho_{i}{ }^{0} . \mathbf{P}(\mathbf{r})$ no longer equals $-\chi \nabla \psi$. Instead it can be written as ${ }^{11,19}$

$$
\mathbf{P}(\mathbf{r})=\mathbf{P}_{0}(\mathbf{r})-\chi_{e}(\mathbf{r}) \nabla\left(\psi-\psi_{0}\right),
$$

where $\mathbf{P}_{0}(\mathbf{r})$ and $\psi_{0}$ are quantities characterizing the nonequilibrium state of the medium surrounding the particles $i$, and where $\chi_{e}$ is the dielectric electronic susceptibility of the medium. The terms $\mathbf{P}_{0}$ and $\psi_{0}$ are independent of all $\rho_{i}{ }^{0}$ and $\rho_{i}{ }^{\text {in }}$. On equating the molecular and continuum particle model expressions for various quantities, as in the earlier section on equivalence of the two models, this environmental term cancels, just as it did in the equilibrium case, so that all equations in that section and in the pertinent discussion given in Appendix I remain unaffected. [Indeed, $\mathbf{P}(\mathbf{r})$ nowhere appears explicitly in those sections.] Similarly, an examination of Appendices II, III, IV, and VII reveals an absence of $\mathbf{P}(\mathbf{r})$, so that these proofs are unaffected also. In Appendix V, $\chi(\mathbf{r})$ appears in Eq. (A24). However, when the last term in (A24) is replaced by its nonequilibrium equivalent

\footnotetext{
${ }^{19} \chi_{a}$ is related to $D_{0 D}$, the square of the refractive index of the medium, $4 \pi \chi_{0}=D_{o p}-1$. In an equilibrium polarization system, $\psi=\psi_{0}$ and $\mathbf{P}_{0}=-\chi \nabla \psi_{0}$, so that (17) reduces to the value given earlier for equilibrium polarization systems.
} 
(17), the remaining few lines of the proof proceed as before. Equations (4) and (12) for $F^{\mathrm{pol}}$ no longer apply. The correct expressions are obtained as follows: Let the above $\mathbf{P}_{\mathbf{0}}(\mathbf{r})$ be the polarization that the medium would have if, for its given orientation polarization, it were in electrostatic equilibrium with a charge distribution. The properties of this hypothetical state and charge distribution are designated by a subscript 0. It is then shown in Appendix VIII that the value of $F^{\text {pol }}$ for the molecular-type and continuumtype models of the particles is given by (18) and (19), respectively.

$$
\begin{aligned}
& F^{\mathrm{pol}}=F_{0}^{\mathrm{pol}}+\sum_{i} \int\left(\phi_{i}+\phi_{i_{0}}\right)\left(\rho_{i}{ }^{0}-\rho_{i_{0}}{ }^{0}\right) d \mathbf{r}, \\
& F^{\mathrm{pol}}=F_{0^{\mathrm{pol}}}+\sum_{i} \int\left(\psi+\psi_{0}\right)\left(\rho_{i}{ }^{v}-\rho_{i_{0}}{ }^{v}\right) d \mathbf{r} .
\end{aligned}
$$

The equality of these expressions is then also shown in Appendix VIII, and the proof for this nonequilibrium polarization case is also now complete.

\section{SELECTION OF $\chi_{i}$}

Any choice of $\chi_{i}$ should be consistent with the behavior of the isolated molecule in the simplest applied potential of interest, the one with constant gradient. Examination of, say, Eqs. (A4) and (A5) of Appendix I reveals that $\chi_{i}(\mathbf{r})$ is related to a dielectric constant $D_{i}(\mathbf{r})$ defined by $(20)$.

$$
D_{i}=1+4 \pi \chi_{i}
$$

Since molecule $i$ is at specified nuclear configuration, $\chi_{i}$ or $D_{i}$ is related only to the electronic polarizability of $i$.

Restricting our attention now to piecewise constant $\chi_{i}$ 's, the value of $\chi_{i}$ is readily obtained for a dielectric sphere as follows:

The Green's function is given by (21)..$^{20}$

$$
\begin{aligned}
G\left(\mathbf{r}, \mathbf{r}^{\prime}\right)=\sum_{n=0}^{\infty} \frac{2 n+1}{n D_{i}+n+1} \frac{r^{n}}{r^{\prime n+1}} P_{n}(\cos \gamma) \\
\left(\mathbf{r} \text { in } V_{i}, \quad \mathbf{r}^{\prime} \text { not in } V_{i}\right),
\end{aligned}
$$

where $\gamma$ is the angle between the vectors $\mathbf{r}$ and $\mathbf{r}^{\prime} . \gamma$ is eliminated using the addition theorem for Legendre polynomials. ${ }^{21}$

$$
\begin{aligned}
& P_{n}(\cos \gamma) \\
& \quad=\sum_{m=-n}^{n} \frac{(n-m) !}{(n+m) !} P_{n}^{m}(\cos \theta) P_{n}^{m}\left(\cos \theta^{\prime}\right) \exp \left[i m\left(\phi-\phi^{\prime}\right)\right]
\end{aligned}
$$

$\partial / \partial n_{i}$ is $\partial / \partial r$.

${ }^{20} \mathrm{Cf}$. J. A. Stratton, Electromagnetic Theory (McGraw-Hill Book Company, Inc., New York, 1941), p. 204. Equation (20) there was multiplied by $4 \pi$ to convert to our units.

21 Reference 20, p. 408. Our $\gamma$ is Stratton's $\theta$ on p. 204. $P_{n}^{-m}=$ $[(n-m) ! /(n+m) !] P_{n}{ }^{m}$.
In a uniform field $E_{z}$ along the $z$ axis, $\phi_{i}$ equals $-E_{z} z$ plus an additive constant. Remembering that $z$ equals $r P_{1}(\cos \theta)$, then using (3) and (16) and noting that the second integral in (16) vanishes since every term in (21) satisfies Laplace's equation in $V_{i}$, one finds

$\int \frac{\rho^{\text {in }}}{\left|\mathbf{r}-\mathbf{r}^{\prime}\right|} d \mathbf{r}=\frac{D_{i}-1}{D_{i}+2} a^{3} \frac{P_{1}\left(\cos \theta^{\prime}\right)}{r^{\prime 2}} E_{z} \quad\left(\mathbf{r}^{\prime}\right.$ in $\left.V_{i}\right) ;$

Eq. (22) also has the form of the potential of an induced dipole moment, $\alpha E_{z}$, equal to $\left(D_{i}-1\right)$ $a^{3} E_{z} /\left(D_{i}+2\right)$. Thereby, $D_{i}$ is found to be the solution of $(23)$ :

$$
D_{i}=\left(a^{3}+2 \alpha\right) /\left(a^{3}-\alpha\right) .
$$

The same value of $\alpha$ is found if one sets $\varphi_{i}=-E_{x} x$ or $-E_{y} y$ so $\alpha$ is isotropic.

Using the Clausius-Mosotti equation, the square of the refractive index $n$ of a closely packed fluid composed of $i$ 's and having negligible free volume is given by the same equation. Hence, $D_{i}$ may be obtained from $n^{2}$ for such a medium: $D_{i} \cong n^{2}$. As remarked earlier, once $D_{i}$ is selected, its value is independent of the other particles present, of their concentration, and of the external fields, to the approximation that these variables do not alter $V_{i}$.

In the case of ellipsoidal particles, the Green's function can be expressed in terms of ellipsoidal harmonics. The leading term is of particular interest, it being the only term when the potential has a constant gradient. In a uniform external field where $\varphi_{i}(r)=-E_{x} x$, one finds ${ }^{22}$

$$
\begin{aligned}
\int \frac{\rho^{\text {in }}\left(\mathbf{r}^{\prime}\right)}{\left|\mathbf{r}-\mathbf{r}^{\prime}\right|} d \mathbf{r}^{\prime}= & \frac{a b c\left(D_{i}-1\right) E_{x} x}{2+a b c\left(D_{i}-1\right) L_{x}} \\
& \times \int_{\xi}^{\infty} \frac{d s}{\left[\left(s+a^{2}\right)^{3}\left(s+b^{2}\right)\left(s+c^{2}\right)\right]^{3}},
\end{aligned}
$$

where $a, b$, and $c$ are the lengths of the semiprincipal axes $(a>b>c), \xi$ is the solution of (25), and $L_{x}$ is given by (26):

$$
\begin{aligned}
& \frac{x^{2}}{a^{2}+\xi}+\frac{y^{2}}{b^{2}+\xi}+\frac{z^{2}}{c^{2}+\xi}=1 \quad\left(\xi>-c^{2}\right), \\
& L_{x}=\int_{0}^{\infty} \frac{d s}{2\left[\left(s+a^{2}\right)^{3}\left(s+b^{2}\right)\left(s+c^{2}\right)\right]^{1}} .
\end{aligned}
$$

If the induced charge density had only a dipolar contribution, say along the $x$ axis, the density would exert a potential $x / r^{3}$ times a constant. Only when $a$, $b$, and $c$ are equal is (24) of such a simple form. Hence, a potential with a constant gradient induces not only a dipole but also higher multipoles as well. At large $r$ (hence, at large $\xi$ ), the leading term in (24) is that

${ }^{22}$ Reference 20 , p. 213. In Eq. (38) there, one subtracts $\phi_{0}$ to obtain the potential due to the induced charge. This $\phi_{0}$ is our $\phi_{i}$. 
due to the induced dipole $\alpha_{x} E_{x}$. Noting that for large $\xi$ the integral in (24) approaches $\frac{2}{3} \xi_{\xi}^{3}$, and setting (24) equal to $\alpha_{x} E_{x} x / r^{3}$, one finds that $D_{i}$ must satisfy (27):

$$
\alpha_{x}=\frac{2}{3} a b c\left(D_{i}-1\right) /\left[2+a b c\left(D_{i}-1\right) L_{x}\right] .
$$

Similarly, $D_{i}$ is also the solution of the corresponding equation with $\alpha_{x}$ and $L_{x}$ replaced by $\alpha_{y}$ and $L_{y}$, and of the corresponding equation with $\alpha_{x}$ and $L_{x}$ replaced by $\alpha_{z}$ and $L_{z}{ }^{23}$ A check on the consistency of the model is obtained by seeing if the $D_{i}$ estimated from each of the three equations is approximately the same.

\section{ERROR OF INDUCED DIPOLE APPROXIMATION FOR A SPHERE}

As noted earlier, the approximation of regarding the induced charge distribution as being an induced dipole at a point in a molecule will break down when the molecule becomes sufficiently large or the field sufficiently nonuniform. In fact, this breakdown has permitted the estimate of molecular size through lightscattering experiments. ${ }^{8,24}$

The error in making the induced dipole approximation is easily estimated for the case of the interaction of a charge with a dielectric sphere, the counterpart of the interaction of an ion with a large spherical molecule. The work required to bring a unit charge and a dielectric sphere from infinity to a distance $R$ apart is given by $(28)^{25}$ :

$$
w(R)=-\frac{\left(D_{i}-1\right)}{2 R^{2}} a \sum_{n=1}^{\infty} \frac{n}{n+1+n D_{i}}(a / R)^{2 n} .
$$

The $n=1$ term is the dipole contribution. If in the other terms one roughly sets $n+1 \cong n$, the ratio of the $n=1$ term to the sum of all remaining term is $\left[\left(D_{i}+1\right) /\left(D_{i}+2\right)\right]\left[(R / a)^{2}-1\right]$. Evidently, even when $R \simeq 2 a$, the correction to the induced dipole approximation is not negligible. When $R$ approaches $a$, a situation which corresponds to an ion approaching an appreciably larger molecule, the error is considerable. If this approach occurs in the solution, and if the surrounding solvent is treated as a dielectric continuum of dielectric constant $D$, the corresponding equation for $w(R)$ is also available $e^{25}$ and is obtained from (28) by replacing $D_{i}$ by $D_{i} / D$. As before, the ratio of the induced dipole term $(n=1)$ to the remainder is about $\left[\left(D_{i}+D\right) /\left(D_{i}+2 D\right)\right]\left[\left(R / a^{2}-1\right]\right.$, which, as before, can be small!

\footnotetext{
${ }^{23} L_{y}$ is $L_{x}$ with $a$ and $b$ interchanged and $L_{z}$ is $L_{x}$ with $a$ and $c$ interchanged. We note that $L_{x}+L_{y}+L_{z}=1$. The approximate values of the $L$ 's are given by $L_{x}: L_{y}: L_{z}=1 / a: 1 / b: 1 / c$.

${ }_{24}$ When the ratio of particle size to wavelength is of the order of 0.1 , a dissymmetry occurs in the scattered light. Estimates of molecular size have been made thereby, particularly in the "Rayleigh-Gans" region, ${ }^{8}$ using in effect the continuum model.

${ }^{25}$ J. G. Kirkwood, Ref. 5. In his Eq. (76) we set $e_{1}=0, D=1$, $Z_{k \mathrm{e}}=1$.
}

\section{POSSIBLE EXPERIMENTAL TEST OF THE MODEL. FOR INDUCED CHARGE}

The inadequacy of an induced point dipole model for light-scattering experiments with very large molecules has been mentioned earlier. Similar experiments for smaller molecules, $20 \AA$ in diameter say, would require light of wavelength of the order of $200 \AA$ or less. This wavelength is not easily accessible, and also the electronic response would differ considerably from that for static fields. An estimate has been made of the contribution of induced quadrupoles in the case of $x$-ray scattering from small ions in crystals. ${ }^{26}$ In this particular case the effects appears to be small.

The study of the induced point dipole approximation by electron scattering also offers various experimental and theoretical difficulties. To make the wavelength appreciably large compared with particle size $\left[\lambda=(150 / V)^{t} \AA\right]$ an unusually low electron energy is needed. In addition, any penetration of the electron cloud of the scattering molecule by the incident electron would also contribute other terms to the interaction energy. Indeed, in experiments at slightly higher energies ( $\sim 1 \mathrm{eV}$ or more) such penetration terms have been the ones used, in conjunction with some rough polarization correction, to explain the scattering patterns. $^{27}$

To increase the wavelength considerably and so investigate the possible breakdown of the approximation with particles of appreciable size, one might study instead the scattering of ions by these molecules. A sodium ion of $2 \mathrm{eV}$ kinetic energy has a $\lambda$ of about $150 \AA$. Equations relating scattering to interaction potential, say, in the WKBJ approximation, have been described ${ }^{2 s}$ and could be applied to the present problem. The total interaction potential is usually taken to be the sum of the polar term, a dispersion term, and for shorter distances a repulsive term. For large $r$ only. the first two need to be considered..$^{29}$ Certain possible contributions of an inelastic nature, such as charge transfer or electronic excitation, can be minimized by use of ions of low electron affinity (e.g., monovalent alkali metal cations) and of low energy.

\section{ACKNOWLEDGMENTS}

The writer would like to acknowledge the support of this research by the U. S. Atomic Energy Commis-

${ }^{26} \mathrm{H}$. Honl, Z. Physik 84, 1 (1933); cf. discussion in R. W. James, The Optical Principles of the Diffraction of $X$ Rays (G. Bell and Sons, Ltd., London, 1950), pp. 161-167.

${ }_{2 \pi}$ Cf. N. F. Mott and H. S. W. Massey, The Theory of Atomic Collisions (Oxford University Press, London, 1950), 2nd ed.

${ }^{28}$ K. W. Ford and J. A. Wheeler, Ann. Phys. N.Y. 7, 259, 289 (1959); R. P. Marchi and C. R. Mueller, J. Chem. Phys. 36, 1100 (1962); R. B. Bernstein, ibid. 36, 1403 (1962) [who gives expressions for an interaction potential energy of the form $f f(r / \sigma)$; $\mathrm{Eq} .(30)$ is of this form].

${ }_{29}$ Cf. "Scattering of Atoms": R. B. Bernstein, J. Chem. Phys. 34, 364 (1961) ; E. W. Rothe and R. B. Bernstein, ibid. 31, 1619 (1959). 
sion, the National Science Foundation, and the Alfred $P$. Sloan Foundation. A portion of this research and that of Part I was performed while the writer was a National Science Foundation Senior Post-Doctoral Fellow at Courant Institute of Mathematical Sciences, New York University.

\section{APPENDIX I: SOLUTION OF EQUATION (A1) IN TERMS OF (A2) AND VICE VERSA}

If $Y$ and $W$ denote any functions for which the pertinent operations are defined, it is shown that, given Eq. (A1), (A2) follows, and conversely.

$$
\begin{aligned}
& Y\left(\mathbf{r}^{\prime}\right)=W\left(\mathbf{r}^{\prime}\right)+\int \chi_{i}(\mathbf{r}) \nabla W(\mathbf{r}) \cdot \nabla r^{-1} d \mathbf{r}, \\
& W\left(\mathbf{r}^{\prime}\right)=Y\left(\mathbf{r}^{\prime}\right)- \int Y(\mathbf{r}) \chi_{i}(\mathbf{r}) \frac{\partial G_{i}}{\partial n_{i}}\left(\mathbf{r}^{\prime}, \mathbf{r}\right) d S_{i} \\
&+\int Y(\mathbf{r}) \nabla \cdot \chi_{i}(\mathbf{r}) \nabla G_{i}\left(\mathbf{r}^{\prime}, \mathbf{r}\right) d \mathbf{r},
\end{aligned}
$$

where $G_{i}\left(\mathbf{r}^{\prime}, \mathbf{r}\right)$ is a Green's function, the solution of Eq. (A3), and also the potential at a point $\mathbf{r}^{\prime}$ in a system having a unit charge at $\mathbf{r}$ and a dielectric susceptibility function $\chi_{i}(\mathbf{r})$

$$
G_{i}\left(\mathbf{r}^{\prime}, \mathbf{r}\right)=r^{-1}-\int \chi_{i}(\mathbf{t}) \nabla_{i} G_{i}(\mathbf{t}, \mathbf{r}) \cdot \nabla_{t} t^{-1} d \mathbf{t}
$$

where $\nabla_{\mathfrak{l}}$ denotes $\boldsymbol{\nabla}$ expressed in terms of the coordinates of $t, 1 / r$ denotes $1 /\left|\mathbf{r}^{\prime}-\mathbf{r}\right|$, and $1 / t$ denotes $1 /\left|\mathbf{r}^{\prime}-\mathbf{t}\right| . G_{i}\left(\mathbf{r}^{\prime}, \mathbf{r}\right)$ can also be defined as the solution of a differential equation (A4), with boundary condition (A5)..$^{30}$

$$
\boldsymbol{\nabla} \cdot\left[\left(1+4 \pi \chi_{i}\right) \nabla G_{i}\left(\mathbf{r}^{\prime}, \mathbf{r}\right)\right]=-4 \pi \delta\left(\mathbf{r}^{\prime}-\mathbf{r}\right) ;
$$

for all $j$ :

$$
\left.\begin{array}{c}
\left(1+4 \pi \chi_{i}\right) \partial G_{i} / \partial n_{j}=\partial G_{i} / \partial n_{j}{ }^{0} \text { at } S_{j} \\
G_{i} \text { continuous at all } S_{i} ; r G_{i}<\infty \text { when } r=\infty
\end{array}\right\},
$$

where $\partial / \partial n_{j}{ }^{0}$ denotes the limit of $\partial / \partial n_{j}$ as one approaches $S_{i}$ from outside of $V_{j}$. One easily shows in a standard fashion $^{31}$ that $G_{i}\left(\mathbf{r}^{\prime}, \mathbf{r}\right)=G_{i}\left(\mathbf{r}, \mathbf{r}^{\prime}\right)$. In (A5)

${ }^{30}$ Proceeding in the same way as when (10) was obtained from $(9),{ }^{17}$ the rhs of (A3) becomes

$$
\frac{1}{r}-\int \frac{\chi_{i}}{t} \frac{\partial G_{i}}{\partial n_{i}} d S_{i}+\int \frac{\nabla \cdot \chi_{i} \nabla G_{i}}{t} d \mathrm{t}
$$

Operating with $\nabla_{r}, 2$ and applying the results of Ref. 18 (p. 150 and Theorem III, p. 156), (A4) follows. Operating instead with $\partial / \partial n_{j}^{\prime}$ and $\partial / \partial n_{j}{ }^{\prime \prime}$ and using the results on p. 160 and p. 164 (Theorem VI) of Ref. 18, (Aं5) is obtained. $\partial / \partial n_{-}$and $\partial / \partial n_{+}$there are our $\partial / \partial n_{j}$ and $\partial / \partial n_{j}{ }^{0}$, respectively. There is a typographical error in one of the equations on p. $160\left(r^{-8}\right.$ should be replaced by $r^{-1}$ ).

${ }_{31}$ P. M. Morse and H. Feshbach, Methods of Theoretical Physics (McGraw-Hill Book Company, Inc., New York, 1953). when $j \neq i, \chi_{i}(\mathbf{r})=0$ and so $\partial G_{i} / \partial n$, equals $\partial G_{i} / \partial n_{j}{ }^{0}$ for $j \neq i$.

We shall also use the relation

$$
\nabla^{2}(1 / r)=-4 \pi \delta\left(\mathbf{r}-\mathbf{r}^{\prime}\right)
$$

In both proofs in this Appendix we apply the identity (A8), obtained as follows:

$W$ (or indeed any function) can be written in the form of an identity (A7), because of (A4) and (A5) :

$$
\begin{aligned}
W\left(\mathbf{r}^{\prime}\right)=\int W & (\mathbf{r}) \delta\left(\mathbf{r}-\mathbf{r}^{\prime}\right) d \mathbf{r} \\
= & -\frac{1}{4 \pi} \int W \nabla \cdot\left(1+4 \pi \chi_{i}\right) \nabla G_{i} d \mathbf{r} \\
& +\sum_{j} \int \frac{W}{4 \pi}\left\{\left(1+4 \pi \chi_{i}\right) \frac{\partial G_{i}}{\partial n_{i}}-\frac{\partial G_{i}}{\partial n_{j}^{0}}\right\} d S_{j} .
\end{aligned}
$$

[We have added the term involving surface integrals, a permissible step since it vanishes because of (A5).] The collection of the surface integrals is, by Gauss' theorem, ${ }^{12}$ equal to

$$
\frac{1}{4 \pi} \int \nabla \cdot\left\{W\left(1+4 \pi \chi_{i}\right) \nabla G_{i}\right\} d \mathbf{r},
$$

which on performing the $\nabla \cdot$ operation yields two integrals, one of which cancels the first one in (A7). Hence

$$
W\left(\mathbf{r}^{\prime}\right)=\frac{1}{4 \pi} \int\left(1+4 \pi \chi_{i}\right) \nabla W \cdot \nabla G_{i} d \mathbf{r} .
$$

This integral can be rewritten as

$$
\int \nabla \cdot G_{i}\left(1+4 \pi \chi_{i}\right) \nabla W d \mathbf{r}-\int G_{i} \nabla \cdot\left(1+4 \pi \chi_{i}\right) \nabla W d \mathbf{r} .
$$

Using Gauss' theorem one then finally obtains the identity,

$$
\begin{aligned}
W\left(\mathbf{r}^{\prime}\right)=- & \frac{1}{4 \pi} \int G_{i} \nabla \cdot\left(1+4 \pi \chi_{i}\right) \nabla W d \mathbf{r} \\
& +\sum_{j} \frac{1}{4 \pi} \int G_{i}\left\{\left(1+4 \pi \chi_{i}\right) \frac{\partial W}{\partial n_{j}}-\frac{\partial W}{\partial n_{j}{ }^{0}}\right\} d S_{j} .
\end{aligned}
$$

Proof that Eq. (A2) for W is the Solution of Eq. (A1)

We first convert the integral in (A1) to

$$
\int \nabla \cdot\left(\frac{\chi_{i} \nabla W}{r}\right) d \mathbf{r}-\int \frac{\nabla \cdot \chi_{i} \nabla W}{r} d \mathbf{r}
$$

and then convert the former to the surface integral

$$
\int \frac{\chi_{i}}{r} \frac{\partial W}{\partial n_{i}} d S_{i}
$$

(Gauss' theorem). One may then obtain Eqs. (A9) and (A10) from (A1) in a way identical to that em- 
ployed to obtain (A4) and (A5) from (A3), by operating separately on (A1) with $\nabla_{r^{\prime}}{ }^{2}, \partial / \partial n_{j}{ }^{\prime}$, and $\partial / \partial n_{j}{ }^{0 \prime}$.

for all $j$ :

$$
\boldsymbol{\nabla} \cdot\left(1+4 \pi \chi_{i}\right) \nabla W=\nabla^{2} Y
$$

$$
\left(1+4 \pi \chi_{i}\right) \frac{\partial W}{\partial n_{j}}-\frac{\partial W}{\partial n_{j}{ }^{0}}=\frac{\partial Y}{\partial n_{j}}-\frac{\partial Y}{\partial n_{j}{ }^{0}} \quad\left(\text { at } S_{j}\right) .
$$

Inserting (A9) and (A10) into the identity (A8) and reversing the procedure used to obtain (A8) from (A7) (two integrations by parts and two applications of Gauss' theorem), one finds

$W\left(\mathbf{r}^{\prime}\right)=-4 \pi^{-1}\left[\int Y \nabla^{2} G_{i} d \mathbf{r}-\int Y\left(\frac{\partial G_{i}}{\partial n_{i}}-\frac{\partial G_{i}}{\partial n_{i}{ }^{0}}\right) d S_{i}\right]$.

Adding and subtracting,

$$
-\int Y \nabla \cdot \frac{\left(4 \pi \chi_{i} \nabla G_{i}\right)}{4 \pi} d \mathbf{r}+\int Y \frac{4 \pi \chi_{i}}{4 \pi} \frac{\partial G_{i}}{\partial n_{i}} d S_{i}
$$

and applying Eqs. (A4) and (A5), one obtains the rhs of (A2).

\section{Proof that Eq. (A1) for $\mathrm{Y}$ is the Solution of Eq. (A2)}

Equation (A2) can be written as (A11) by reversing the last step of the preceding section. But since $W$ is also given by the identity (A8), the rhs's of these equations can be equated, and, after some manipulation, the result can be written as

$$
\int G_{i} \rho^{u} d \mathbf{r}+\sum_{j} \int G_{i} \sigma_{j}^{u} d S_{j}=0,
$$

where $\rho^{u}$ and $\sigma_{j}{ }^{u}$ are

$$
\begin{aligned}
\rho^{u} & =\nabla \cdot\left\{\left(1+4 \pi \chi_{i}\right) \nabla W-\nabla Y\right\}, \\
\sigma_{i}^{u} & =-\left(1+4 \pi \chi_{i}\right) \frac{\partial W}{\partial n_{j}}-\frac{\partial W}{\partial n_{j}^{0}}+\frac{\partial Y}{\partial n_{j}}-\frac{\partial Y}{\partial n_{j}^{0}} .
\end{aligned}
$$

[Use was made of (A5) : $\partial G_{i} / \partial n_{j}=\partial G_{i} / \partial n_{j}{ }^{0}$ when $j \neq i$.]

From the meaning of the Green's function, we conclude from Eq. (A12) that in a medium in which the dielectric susceptibility is $x_{i}$ in $V_{i}$ and zero everywhere else, a system with volume charge density $p^{u}$ and surface charge densities $\sigma_{j}{ }^{u}$ has a potential which vanishes identically. Hence $\rho_{j}{ }^{u}$ and all $\sigma_{j}{ }^{u}$ must vanish everywhere. From the expressions for $\rho_{j}{ }^{u}$ and $\sigma_{j}{ }^{u}$ Eqs. (A9) and (A10) then immediately follow. From the latter, (A1) can be deduced by multiplying (A9) by $1 / r$, integrating over $r$, integrating by parts, appplying Gauss' theorem and finally using (A10) and (A6).

APPENDIX II: SOLUTION OF EQ. (16) FOR $A_{i}$

Since the induced charge density may have both volume and surface contributions, we may write $A_{i}$ without loss of generality as in (A13),

$$
A_{i}=A_{i}{ }^{\rho}+A_{i}{ }^{g} \delta\left(n_{i}\right),
$$

and solve for $A_{i}{ }^{p}$ and $A_{i}{ }^{8}$.
Introducing (A13) into (16), one may obtain from (16) Eqs. (A14) and (A15) in the same way that (A4) and (A5) were obtained from (A3), by operating separately with $\nabla_{r^{\prime}}{ }^{2}, \partial / \partial n_{i}{ }^{\prime}$, and $\partial / \partial n_{i}^{{ }^{\prime}}$, etc.

$-4 \pi A_{i}{ }^{\rho} \phi_{i}=\nabla_{r^{2}}\left[\int \phi_{i} \nabla \cdot\left(\chi_{i} \nabla G_{i}\right) d \mathbf{r}-\int \phi_{i} \chi_{i} \frac{\partial G_{i}}{\partial n_{i}} d S_{i}\right]$,

$4 \pi A_{i}{ }^{s} \phi_{i}=\left(\frac{\partial}{\partial n_{i}{ }^{\prime}}-\frac{\partial}{\partial n_{i}{ }^{\prime}}\right)\left[\int \phi_{i} \nabla \cdot \chi_{i} \nabla G_{i} d \mathbf{r}-\int \phi_{i} \chi_{i} \frac{\partial G_{i}}{\partial n_{i}} d S_{i}\right]$.

(We used the fact that $\int A_{i}{ }^{p} \phi_{i} d \mathbf{r} / r$ has a continuous normal gradient at $S_{i}$.)

Operator equations for $A_{i}{ }^{p}$ and $A_{i}^{s}$ are obtained from (A14) and (A15) by omitting $\phi_{i}$. We see that both $A_{i}{ }^{p}$ and $A_{i}{ }^{s}$ are operators which depend only on $\chi_{i}$ and on the geometry of $i$. ( $\chi_{i}$ vanishes outside of $V_{i}$ )

\section{APPENDIX III: SOLUTION FOR $\rho_{i}{ }^{0}$}

We first note from Eq. (8) that $\psi_{\text {is }}$ can be expressed in terms of $\rho_{i}{ }^{v}, \chi_{i}$, and the geometry of $i$, using the $G_{i}\left(\mathbf{r}, \mathbf{r}^{\prime}\right)$ of Appendix I: Multiplying (A3) by $\rho_{i}{ }^{v}(\mathbf{r})$, integrating over $\mathbf{r}$, and interchanging the integration order, one obtains an equation for $\int G_{i} \rho_{i}{ }^{*} d \mathbf{r}$ similar to Eq. (8) for $\psi_{\text {is. }}$ By the uniqueness theorem for potentials $^{18}$ we therefore have

$$
\psi_{\mathrm{is}}=\int G_{i}\left(\mathbf{r}^{\prime}, \mathbf{r}\right) \rho_{i}{ }^{v} d \mathbf{r} .
$$

From the fact that in the continuum model of a particle $\psi_{\text {is }}$ is given by an equation similar to (6), but with $\rho_{i}^{0}$ replaced with $\rho_{\text {is }}$, and from the fact that $\rho_{\text {is }}$ is given by (9), we have

$$
\rho_{i}^{0}=\rho_{i}{ }^{v}+\nabla \cdot\left(\chi \cdot \nabla \psi_{\mathrm{is}}\right)-\delta\left(n_{i}\right) \chi_{i}\left(\partial \psi_{\mathrm{is}} / \partial n_{i}\right),
$$

where $\psi_{\text {is }}$ has the value given by Eq. (A16). Inserting (A16) into Eq. (A17), we see that $\rho_{i}{ }^{0}$ depends only on $\chi_{i}, \rho_{i}{ }^{v}$, and the geometry of $i$.

\section{APPENDIX IV: EQUIVALENCE OF EXPRESSIONS FOR $\psi_{\text {is }}$ FOR THE TWO MODELS}

Introducing $\rho_{i}^{0}$ given by Eqs. (A16) and (A17) into Eq. (6) for $\psi_{\mathrm{is}}{ }^{M}$ ( $M$ denotes molecular model), one obtains, after an integration by parts and after interchanging the order of integration,

$\psi_{\mathrm{is}}{ }^{M}\left(\mathbf{r}^{\prime}\right)=\int \rho_{i}{ }^{\nu}(\mathbf{t}) d \mathbf{t}\left[t^{-1}-\int \chi_{i} \nabla G_{i}(\mathbf{r}, \mathbf{t}) \cdot \nabla r^{-1} d \mathbf{r}\right]$.

Introducing Eq. (A3) for $G_{i}$, the rhs of Eq. (A18) becomes

$$
\int \rho_{i}^{v}(\mathbf{t}) G_{i}\left(\mathbf{r}^{\prime}, \mathbf{t}\right) d \mathbf{t}
$$


which is also the value of $\psi_{\text {is }}$ for the continuum model [cf. Eq. (A16)].

\section{APPENDIX V: EQUIVALENCE OF EXPRESSIONS FOR $\psi$ FOR THE TWO MODELS}

From the values of $A_{i}{ }^{p}$ and $A_{i}{ }^{s}$ derived in Appendix II, expressed in terms of properties of the continuum model, we first obtain Eq. (A19):

Using (A13) to operate on an arbitrary function $V$, then dividing $A_{i} V$ by $r$, integrating over $\mathbf{r}$, and introducing (A14) and (A15) (with $\phi_{i}$ replaced by the arbitrary function $V$ ), one obtains an expression for $\int A_{i} V d \mathbf{r} / r$. Integrating by parts and applying Gauss' theorem twice and using (A6), one finds

$$
\int \frac{A_{i} V}{r} d \mathbf{r}=\int V \boldsymbol{\nabla} \cdot\left(\chi_{i} \nabla G_{i}\right) d \mathbf{r}-\int V \chi_{i} \frac{\partial G_{i}}{\partial n_{i}} d S_{i}
$$

However, from Eqs. (2), (3), (5b), and (6), one finds

$$
\phi_{i}{ }^{M}\left(\mathbf{r}^{\prime}\right)=\psi^{M}-\psi_{\mathrm{is}}{ }^{M}-\int \frac{A_{i} \phi_{i}{ }^{M}}{r} d \mathbf{r},
$$

which when combined with (A19) (with $V$ now replaced by $\phi_{i}{ }^{M}$ ) yields

$$
\begin{array}{r}
\psi^{M}\left(\mathbf{r}^{\prime}\right)-\psi_{\mathrm{is}}{ }^{M}\left(\mathbf{r}^{\prime}\right)=\phi_{i}{ }^{M}+\int \phi_{i}{ }^{M} \nabla \cdot\left(\chi_{i} \nabla G_{i}\right) d \mathbf{r} \\
-\int \phi_{i}{ }^{M} \chi_{i} \frac{\partial G_{i}}{\partial n_{i}} d S_{i} .
\end{array}
$$

This equation may be inverted to give (A22), by applying the second proof in Appendix I [deduction of (A1) from (A2), with $Y=\phi_{i}{ }^{M}$ and $\left.W=\psi^{M}-\psi_{\text {is }}{ }^{M}\right]$,

$$
\phi_{i}{ }^{M}=\psi^{M}-\psi_{\mathrm{is}}{ }^{M}+\int \chi_{i} \nabla\left(\psi^{M}-\psi_{\mathrm{is}}{ }^{M}\right) \cdot \nabla r^{-1} d \mathbf{r}
$$

From (A20) and (A22), (A23) follows:

$$
\int \frac{A_{i} \phi_{i}{ }^{M}}{r} d \mathbf{r}=-\int \chi_{i} \nabla\left(\psi^{M}-\psi_{\mathrm{is}}{ }^{M}\right) \cdot \nabla r^{-1} d \mathbf{r}
$$

Introducing (2), (3), and (A23), Eq. (5a) for $\psi^{M}$ becomes

$$
\begin{aligned}
\psi^{M}\left(\mathbf{r}^{\prime}\right)=\sum_{i}\left\{\int \frac{\rho_{i}^{0}}{r} d \mathbf{r}-\int \chi_{i} \nabla\left(\psi^{M}-\psi_{\mathrm{is}}{ }^{M}\right)\right. & \left.\nabla r^{-1} d \mathbf{r}\right\} \\
& -\int \chi \nabla \psi^{M} \cdot \nabla r^{-1} d \mathbf{r},
\end{aligned}
$$

on setting $\mathbf{P}(\mathbf{r})$ equal to $-\chi \nabla \psi^{M}$. The first integral on the rhs equals $\psi_{\text {is }}{ }^{M}$, but this equals the continuum $\psi_{\text {is }}$ (Appendix IV), $\int\left(\rho_{i}^{V}-\chi_{i} \nabla \psi_{\text {is }} \cdot \nabla\right) 1 / r d \mathbf{r}$. Making this substitution, one obtains

$$
\psi^{M}=\sum_{i} \int\left[\frac{\rho_{i}{ }^{V}}{r}-\chi_{i} \nabla \psi^{M} \cdot \nabla r^{-1}\right] d \mathbf{r}-\int \chi \nabla \psi^{M} \cdot \nabla r^{-1} d \mathbf{r} .
$$

This equation is the same as that satisfied by $\psi$ for the continuum model [Eq. (7)]. By the uniqueness theorem for potentials, they are therefore equal.

\section{APPENDIX VI: EQUIVALENCE OF EXPRESSIONS FOR Frol FOR THE TWO MODELS}

Introducing Eq. (A22) for $\phi_{i}{ }^{M}$ into Eq. (4) for $F_{M}{ }^{\mathrm{pol}}$, interchanging the order of integration, and using Eq. (6), one obtains

$$
\begin{aligned}
& F_{M^{\mathrm{pol}}=\frac{1}{2} \sum_{i}\left[\int\left(\psi^{M}-\psi_{\mathrm{is}}{ }^{M}\right) \rho_{i}{ }^{0} d \mathbf{r}\right.} \\
& \left.\quad+\int \chi_{i} \nabla\left(\psi^{M}-\psi_{\mathrm{is}}{ }^{M}\right) \cdot \nabla \psi_{\mathrm{is}} d \mathbf{r}\right]
\end{aligned}
$$

Introducing Eq. (A17) for $\rho_{i}{ }^{0}$, one obtains (A26), after integrating the last term in (A25) by parts and remembering that $\chi_{i}=0$ outside of $V_{i}$ :

$$
F_{M}{ }^{\mathrm{pol}}=\frac{1}{2} \sum_{i} \int\left(\psi^{M}-\psi_{\mathrm{is}}{ }^{M}\right) \rho_{i}{ }^{v} d \mathbf{r}
$$

Since $\psi^{M}$ and $\psi_{\text {is }}{ }^{M}$ are the same as $\psi$ and $\psi_{\text {is }}$ for the continuum models (Appendices IV and V), this expression is identical with Eq. (12) for the continuum value of $F^{\mathrm{pol}}$.

\section{APPENDIX VII: EQUTVALENCE OF THE EXPRESSIONS FOR $\rho_{i}$}

Because of Eq. (A17) the expressions of the two models for $\rho_{\text {is }}$ (i.e., for $\rho_{i}{ }^{0}$ ) are made automatically the same by choosing $\rho_{i}{ }^{v}$ to satisfy (A17), for any assigned $\rho_{i}^{0}(\mathbf{r}), \chi_{i}(\mathbf{r})$, and geometry of $i$.

The expression for $\rho_{i}$ for the molecular model is given by (A27), which follows from (2) and (3):

$$
\rho_{i}{ }^{M}(\mathbf{r})=\rho_{i}{ }^{0}(\mathbf{r})+A_{i} \phi_{i}{ }^{M} .
$$

Because of the $1: 1$ correspondence between any function $\rho_{i}(\mathbf{r})$ and the function $\int \rho_{i}(\mathbf{r}) / r d \mathbf{r}$, it suffices to prove equivalence of the latter for the two models.

From Eqs. (A27), (A17), and (A23) one obtains, after using Gauss' theorem and recalling the boundary conditions on $\psi_{\text {is, }}$,

$$
\int \frac{\rho_{i}{ }^{M}}{r} d \mathbf{r}=\int \frac{\rho_{i}^{v}}{r} d \mathbf{r}-\int \chi_{i} \nabla \psi^{M} \cdot \nabla r^{-1} d \mathbf{r} .
$$

The rhs of this equation is, with $\psi^{M}$ replaced by the $\psi$ for the continuum model of a molecule, equal to $\int \rho_{i}{ }^{C} / r d \mathbf{r}$ (where $\rho_{i}{ }^{C}$ is the $\rho_{i}$ for the continuum model). Since $\psi^{M}$ and $\psi^{C}$ are equal (Appendix V), it then follows from this equation that $\rho_{i}{ }^{M}$ and $\rho_{i}{ }^{C}$ are equal also.

\section{APPENDIX VIII: FDol FOR NONEQUILIBRIUM POLARIZATION SYSTEMS}

Let $\lambda$ be a charging parameter describing the change (at fixed orientation polarization) from the equilibrium polarization state $(\lambda=0)$ to the specified nonequilib- 
rium state $(\lambda=1)$, i.e.,

$$
\rho_{i}{ }^{\lambda}=\rho_{i 0}{ }^{2}+\lambda\left(\rho_{i}{ }^{2}-\rho_{i 0}{ }^{v}\right) \text {. }
$$

$\rho_{i_{0}}{ }^{v}$ and $\rho_{i}{ }^{v}$ are the values of $\rho_{i}{ }^{{ }^{\lambda}}$ at $\lambda=0$ and $\lambda=1$, respectively. Using (7) and (17) we may then write

$$
\begin{aligned}
& \psi^{\lambda}=\sum_{i} \int\left(\frac{\rho_{i}^{i^{\lambda}}}{r}-\chi_{i} \nabla \psi^{\lambda} \cdot \nabla r^{-1}\right) d \mathbf{r} \\
&+\int\left[\mathbf{P}_{0}-\chi_{e} \boldsymbol{\nabla}\left(\psi^{\lambda}-\psi^{0}\right)\right] \cdot \nabla r^{-1} d \mathbf{r},
\end{aligned}
$$

whence

$$
\begin{array}{r}
\psi^{\lambda}-\psi_{0}=\sum_{i} \int\left[\frac{\rho_{i}^{i^{\lambda}}-\rho_{i_{0}}{ }^{p}}{r}-\chi_{i} \nabla\left(\psi^{\lambda}-\psi_{0}\right) \cdot \nabla r^{-1}\right] d \mathbf{r} \\
-\int \chi_{e} \nabla\left(\psi^{\lambda}-\psi^{0}\right) \cdot \nabla r^{-1} d \mathbf{r} .
\end{array}
$$

It follows from (A28) and (A29) that $\left(\psi^{\lambda}-\psi_{0}\right) / \lambda$ and $\psi-\psi_{0}$ satisfy the same equation, so that

$$
\psi^{\lambda}-\psi_{0}=\lambda\left(\psi-\psi_{0}\right)
$$

In virtue of (A17) and the proven equivalence of the charge distributions in the two models and their expressions for the $\psi^{\prime} \mathrm{s}, \rho_{i}{ }^{{ }^{\lambda}}$ obeys an equation similar to (A28):

$$
\rho_{i}^{0^{\lambda}}=\rho_{i_{0}}^{0}+\lambda\left(\rho_{i}^{0}-\rho_{i_{0}}^{0}\right)
$$

Using (A21) for $\phi_{i}{ }^{\lambda}, \phi_{i}$, and $\phi_{i 0}$, then inserting (6), (A30), and (A31) in each case, (A32) follows.

$$
\phi_{i}^{\lambda}-\phi_{i_{0}}=\lambda\left(\phi_{i}-\phi_{i_{0}}\right) \text {. }
$$

For nonequilibrium polarization systems, Eqs. (4) and (11) for $F^{\mathrm{pol}}$ are to be replaced by

$$
\begin{aligned}
& F^{\mathrm{pol}}=F_{0}^{\mathrm{pol}}+\sum_{i} \iint_{\lambda=0}^{1} \phi_{i}^{\lambda} d \rho_{i}^{0} d \lambda d \mathbf{r} \quad \text { (molecular), } \\
& F^{\mathrm{pol}}=F_{0}^{\mathrm{pol}}+\sum_{i} \iint_{\lambda=0}^{1} \psi^{\lambda} d \rho_{i}^{i^{\lambda}} d \lambda d \mathbf{r} \quad \text { (continuum). }
\end{aligned}
$$

Introducing (A30) and (A32), we obtain

(molecular) $F^{\mathrm{pol}}=F_{0}+\frac{1}{2} \sum_{i} \int\left(\phi_{i}+\phi_{i_{0}}\right)\left(\rho_{i}{ }^{0}-\rho_{i_{0}}{ }^{0}\right) d \mathbf{r}$,

(continuum) $F^{p 1}=F_{0}+\frac{1}{2} \sum_{i} \int\left(\psi+\psi_{0}\right)\left(\rho_{i}^{v}-\rho_{i_{0}}{ }^{v}\right) d \mathbf{r}$.

The equivalence proof is now similar to that used in Appendix VI. Equation (A22) is introduced, there being an equation of this type relating $\phi_{i 0}{ }^{M}$ to $\psi_{0}{ }^{M}$ and another of the same type relating $\phi_{i}^{M}$ to $\psi^{M}$. In this way the analog of (A25) is obtained from (A33). After introducing (A17) for $\rho_{i}{ }^{0}$, the analog of (A26) is obtained, and this proves to be the same as (A34).

\title{
Microwave Spectrum of Normal Propyl Chloride
}

\author{
T. N. SARAChMAN* \\ National Bureau of Standards, Washington 25, D.C. \\ (Received 18 March 1963)
}

\begin{abstract}
The microwave spectrum of gaseous normal propyl chloride reveals the presence of two rotational isomers, the trans and gauche conformations. The ground vibrational state rotational constants faund for the more abundant $\mathrm{Cl}^{25}$ species are, for the gauche form, $A=11829.22, B=3322.58, C=2853.06 \mathrm{Mc} / \mathrm{sec}$; for the trans form, $B=2379.7, C=2271.7 \mathrm{Mc} / \mathrm{sec}$. Analysis of quadrupole hyperfine splittings in the spectrum of the gauche form gives the quadrupole coupling constants $\chi_{a \pi}=-19.41 \mathrm{Mc} / \mathrm{sec}, \eta=2.10_{5}$, in the principal axis system of the $\mathrm{Cl}^{35}$ species. Satellite spectra rising from excited vibrational states were also observed: in the gauche form, the first and second excited states of the skeletal torsion were assigned, and another satellite is believed to be from the excited methyl torsion; in the trans form, only the first excited state of the skeletal torsion was assigned. From relative intensity measurements, it is concluded that the energy difference between the trans and gauche isomers is $0 \pm 0.5 \mathrm{kcal} / \mathrm{mole}$.
\end{abstract}

\section{INTRODUCTION}

$\mathbf{T}$ UHE infrared and Raman spectra of gaseous, liquid, and solid normal propyl chloride and bromide, $\mathrm{CH}_{3} \mathrm{CH}_{2} \mathrm{CH}_{2} \mathrm{X}$, have been studied by a number of investigators. ${ }^{1}$ The conclusion drawn from these ex-

* National Research Council-National Bureau of Standards Postdoctoral Research Associate, 1961-1963.

A summary is given by $N$. Sheppard in Advan. Spectry. 1 , 288 (1959). periments is that these molecules exist in the gas phase in two stable conformations of roughly equal energy, trans (the heavy atoms forming a staggered planar chain) and gauche (the $\mathrm{CH}_{2} \mathrm{X}$ group rotated by roughly $120^{\circ}$ from the trans configuration).

A study of the microwave spectrum of normal propyl chloride seemed particularly desirable in view of the well-known sensitivity of rotational transition frequencies to molecular structure. In addition, there is the 